\title{
Le vaccin contre la fièvre jaune: un nouveau traitement anti-tumoral ?
}

Tacien Petithomme, Marion Grard, Jean-François Fonteneau, Nicolas Boisgerault

> La révolution actuelle du traitement des cancers par immunothérapie repose principalement sur l'utilisation d'anticorps monoclonaux inhibiteurs de points de contrôle immunitaire (antiPD-1, anti-PD-L1, anti-CTLA-4, etc.) qui visent à lever l'inhibition des lymphocytes $T$ cytotoxiques capables de cibler et de détruire spécifiquement les cellules tumorales [1]. D'autres types d'immunothérapies anti-tumorales actuellement en développement ont pour objectif d'agir simultanément sur plusieurs mécanismes immunitaires afin d'augmenter l'efficacité observée avec ces anticorps monoclonaux et de limiter l'acquisition de résistances par les cellules tumorales quand un seul de ces mécanismes est ciblé. Dans cette optique, de nombreux laboratoires s'intéressent à l'utilisation de microorganismes dont les propriétés immunogènes naturelles permettent d'activer les cellules immunitaires et de stimuler des réponses dirigées contre les celIules tumorales. C'est notamment le cas de l'immunothérapie oncolytique (Figure 1), qui utilise des virus capables non seulement de détruire spécifiquement les cellules malignes sans endommager les cellules saines, mais aussi d'activer différents acteurs de l'immunité [2]. L'infection des cellules tumorales par ces virus oncolytiques induit une forme de mort cellulaire immunogène qui attire des effecteurs immunitaires et active les cellules dendritiques - chefs d'orchestre de la réponse immunitaire - par reconnaissance de signaux de danger cellulaires (interfé- ron de type I, ATP, etc.) et viraux (ARN double brin, etc.) libérés par les cellules tumorales lysées. Les cellules dendritiques activées sont alors capables de présenter des antigènes tumoraux aux lymphocytes $T$, qui reconnaîtront spécifiquement les cellules tumorales [3]. L'induction de cette réponse immunitaire confèrera idéalement une protection contre la survenue de métastases tumorales et contre d'éventuelles récidives de la tumeur d'origine. Les propriétés immunoactivatrices des virus oncolytiques peuvent en outre être améliorées par l'insertion, dans leur génome, de transgènes thérapeutiques destinés à amplifier la réponse immunitaire dans le microenvironnement tumoral au sein duquel le virus se réplique.

Dans un article récent, Aznar et al. [4] ont utilisé la souche vaccinale 17D du virus de la fièvre jaune (famille des flaviviridae) afin de stimuler cette réponse immunitaire anti-tumorale. Dans un premier temps, ils ont montré que ce virus réplicatif atténué était capable d'infecter et de tuer in vitro plusieurs lignées de cellules tumorales humaines sans détruire les cellules saines, démontrant ainsi son potentiel oncolytique. Dans des modèles animaux de mélanome et de cancer colorectal, l'injection intratumorale du virus 17D permet en outre de retarder la croissance tumorale, avec un effet thérapeutique d'autant plus important que le virus est injecté tôt. Les propriétés thérapeutiques du virus 17D dépendent de l'activation de la réponse immunitaire puisque des tumeurs à distance de celles injectées avec le virus, et
Université de Nantes, Inserm, Centre de recherche en cancérologie et immunologie Nantes-Angers (CRCINA), 8 quai Moncousu, 44000 Nantes, France. nicolas.boisgerault@inserm.fr

dans lesquelles le virus n'est pas détecté, présentent aussi une croissance ralentie. Les auteurs montrent notamment que la réponse antivirale innée (interférons de type I), les lymphocytes T CD8 ${ }^{+}$et les cellules dendritiques $\mathrm{CDIC}^{+}$jouent un rôle central dans cette réponse immunitaire. Si l'effet thérapeutique du virus utilisé en monothérapie était relativement limité, cette étude apporte de nouvelles preuves de l'utilité d'intégrer des virus oncolytiques dans le cadre de protocoles d'immunothérapie puisqu'ils améliorent leur efficacité. Ainsi, les auteurs montrent que la combinaison du virus 17D avec un anticorps agoniste anti-CD137/4-1BB, qui permet une activation plus importante des lymphocytes $T$, induit un contrôle total des tumeurs traitées. Par ailleurs, la combinaison du virus 17D avec un anticorps bloquant anti-PD-1 a un léger effet thérapeutique sur les tumeurs situées à distance et non injectées avec le virus. Ces réponses sont liées à une augmentation de l'infiltrat immunitaire dans les tumeurs injectées avec le virus 17D, infiltrat notamment caractérisé par une augmentation importante du nombre de lymphocytes $\mathrm{T} \mathrm{CD} 8^{+}$cytotoxiques et une diminution spectaculaire du nombre des lymphocytes $T$ CD4 ${ }^{+}$régulateurs. Dans ce contexte, les lymphocytes T CD8 ${ }^{+}$et $C D 4^{+}$ infiltrés présentent un phénotype réactif caractérisé par une augmentation de l'expression de CTLA-4 et une baisse de l'expression de CD137 et de PD-1 à leur surface. Dans les ganglions drainant la tumeur injectée avec le virus et dans les tumeurs situées à distance, les 


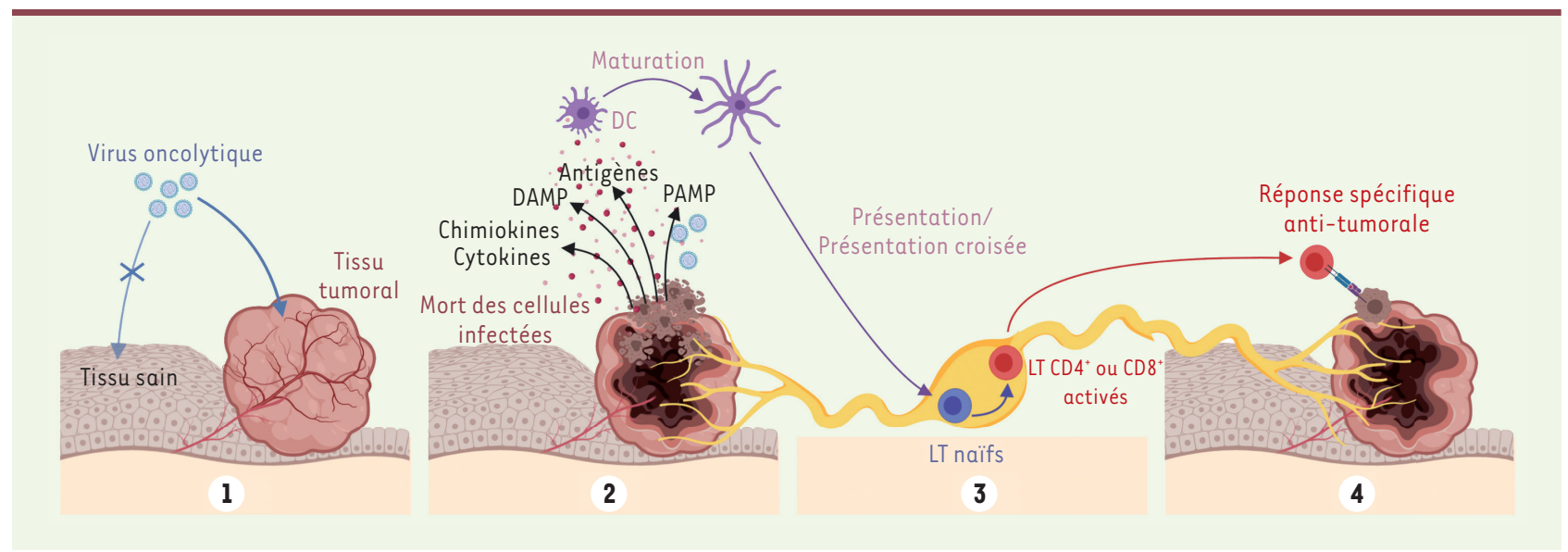

Figure 1. L'immunothérapie oncolytique. Les virus oncolytiques sont des virus réplicatifs infectant préférentiellement les tissus tumoraux (1). En se répliquant, le virus induit la mort des cellules tumorales infectées et la libération de signaux de dangers (DAMP, PAMP, chimiokines, cytokines, etc.), permettant la maturation de cellules dendritiques (dendritic cells, DC), ainsi que la présentation d'antigènes viraux et tumoraux (2). Ces $D C$ migrent dans les organes lymphoïdes secondaires où elles promeuvent la différenciation et la prolifération de lymphocytes T (LT) spécifiques d'antigènes tumoraux (3). Ces LT activés migrent en périphérie et exercent une réponse cytotoxique anti-tumorale (4). DAMP : damage-associated molecular patterns (motifs moléculaires associés aux dégâts cellulaires) ; PAMP : pathogen-associated molecular patterns (motifs moléculaires associés aux microorganismes pathogènes). (Image créée avec BioRender.com).

lymphocytes T et les cellules NK (natural killer) montrent en revanche une augmentation de l'expression de CD137 et de PD-1, ce qui en fait des cibles potentielles pour des anticorps ciblant ces deux marqueurs. Cette étude confirme des résultats obtenus précédemment avec d'autres virus oncolytiques comme I'herpèsvirus T-Vec [5] ou le Newcastle disease virus [6], et valide une nouvelle fois l'idée d'utiliser les virus oncolytiques en combinaison avec des inhibiteurs de points de contrôle immunitaire, en particulier pour traiter des tumeurs dites «froides» du point de vue immunologique, c'est-à-dire peu infiltrées par des cellules immunitaires.

Les résultats publiés par Aznar et al. montrent donc une efficacité relative du virus oncolytique 17D lorsque celuici est utilisé seul comme traitement intra-tumoral, avec des ralentissements de la croissance tumorale, mais pas de régression complète ni de survie à long terme des animaux traités. Ces résultats sont cependant encourageants concernant l'activation de la réponse immunitaire anti-tumorale lorsque le virus est utilisé en combinaison avec des anticorps immunomodulateurs. Des tra- vaux supplémentaires seront nécessaires pour identifier les conditions optimales de traitement (doses utilisées, nombre d'injections, etc.) afin d'augmenter son efficacité thérapeutique, en particulier pour envisager des injections par voie intraveineuse. Ces études complémentaires permettront aussi de mieux situer le virus 17D par rapport aux virus oncolytiques concurrents actuellement soumis à des tests précliniques et cliniques. Du point de vue immunitaire, certains mécanismes restent à élucider, en particulier pour rendre compte de l'observation qu'une pré-immunisation avec le virus 17D avant l'inoculation des tumeurs améliore la réponse immunitaire et son efficacité thérapeutique. Le virus 17D présente en revanche de nombreux avantages qui pourraient faciliter son transfert vers une utilisation clinique. En effet, son profil de toxicité est déjà bien caractérisé puisque cette souche virale est utilisée comme vaccin depuis de nombreuses années et a déjà été injectée chez l'homme par voie sous-cutanée ou intramusculaire dans les régions du monde à risque d'infection par le virus de la fièvre jaune [7]. Cela rappelle le cas des souches vaccinales du virus de la rougeole, dont l'innocuité est démontrée, et qui présentent elles aussi des propriétés oncolytiques naturelles exploitées par plusieurs équipes de recherche [8] jusqu'au stade clinique [9]. L'utilisation possible du virus $17 \mathrm{D}$ après une préimmunisation comporterait un avantage supplémentaire du point de vue de la sécurité, et permettrait notamment d'utiliser des doses thérapeutiques de virus plus importantes. Concernant la production industrielle, les résultats de l'article montrent que le virus produit sur des embryons de poulet - selon le même processus que celui utilisé pour la production du vaccin - conserve son efficacité oncolytique et ses propriétés immunostimulantes, ce qui devrait faciliter son utilisation en virothérapie anti-tumorale.

$\varepsilon$ conclusion, les résultats de l'étude d'Aznar et al. indiquent que le virus oncolytique $17 \mathrm{D}$ est un agent prometteur pour le traitement des cancers. Son efficacité lorsqu'il est utilisé en combinaison avec un anticorps agoniste anti-CD137/4-1BB en fait un candidat intéressant pour les nouvelles stratégies d'immunothérapie ciblant simultanément différents mécanismes immunitaires afin d'amélio- 
rer l'efficacité thérapeutique. D'autres types de combinaisons, voire la modification du virus pour qu'il transporte des transgènes thérapeutiques au sein du microenvironnement tumoral, pourraient permettre d'améliorer encore davantage son potentiel thérapeutique dans les années à venir. $\diamond$

The yellow fever vaccine as a new cancer treatment?

\section{LIENS D'INTÉRÊT}

Les auteurs déclarent n'avoir aucun lien d'intérêt concernant les données publiées dans cet article.

\section{RÉFÉRENCES}

1. Fritz JM, Lenardo MJ. Development of immune checkpoint therapy for cancer.J Exp Med 2019 ; 216 : 1244-54.

2. Bommareddy PK, Shettigar M, Kaufman HL. Integrating oncolytic viruses in combination cancer immunotherapy. Nat Rev Immunol 2018 ; 18 : 498-513.

3. Guillerme J-B, Boisgerault N, Roulois D, et al. Measles virus vaccine-infected tumor cells induce tumor antigen cross-presentation by human plasmacytoid dendritic cells. Clin Cancer Res 2013 ; 19 : 1147-58.

4. Aznar MA, Molina C, Teijeira A, et al. Repurposing the yellow fever vaccine for intratumoral immunotherapy. EMBO Mol Med 2020 ; 12 : el 0375.

5. Ribas A, Dummer R, Puzanov I, et al. Oncolytic virotherapy promotes intratumoral T cell infiltration and improves anti-PD-1 immunotherapy. Cell 2017 ; 170 : 1109-19.e10.
6. Zamarin D, Holmgaard RB, Subudhi SK, et al. Localized oncolytic virotherapy overcomes systemic tumor resistance to immune checkpoint blockade immunotherapy. Sci Transl Med 2014 ; 6 : 226 ra32.

7. Barrett ADT. Yellow fever live attenuated vaccine: $A$ very successful live attenuated vaccine but still we have problems controlling the disease. Vaccine 2017 ; $35: 5951-5$

8. Achard C, Boisgerault N, Delaunay T, et al. Sensitivity of human pleural mesothelioma to oncolytic measles virus depends on defects of the type I interferon response. Oncotarget $2015 ; 6: 44892-904$.

9. Galanis $\varepsilon$, Atherton PJ, Maurer MJ, et al. Oncolytic measles virus expressing the sodium iodide symporter to treat drug-resistant ovarian cancer. Cancer Res $2015 ; 75: 22-30$. 